\title{
Formation flying performances simulator for the shadow position sensors of the ESA PROBA-3 mission
}

Gerardo Capobianco, Francesco Amadori, Silvano Fineschi, Alessandro Bemporad, Marta Casti, et al.

Gerardo Capobianco, Francesco Amadori, Silvano Fineschi, Alessandro Bemporad, Marta Casti, Davide Loreggia, Vladimiro Noce, Maurizio Pancrazzi, Federico Landini, Cedric Thizy, Raphael Rougeot, Damien Galano, Jorg Versluys, "Formation flying performances simulator for the shadow position sensors of the ESA PROBA-3 mission," Proc. SPIE 11852, International Conference on Space Optics - ICSO 2020, 118526P (11 June 2021); doi: 10.1117/12.2600310

SPIE Event: International Conference on Space Optics - ICSO 2021, 2021, Online Only 


\section{International Conference on Space Optics-ICSO 2020}

Virtual Conference

30 March-2 April 2021

Edited by Bruno Cugny, Zoran Sodnik, and Nikos Karafolas
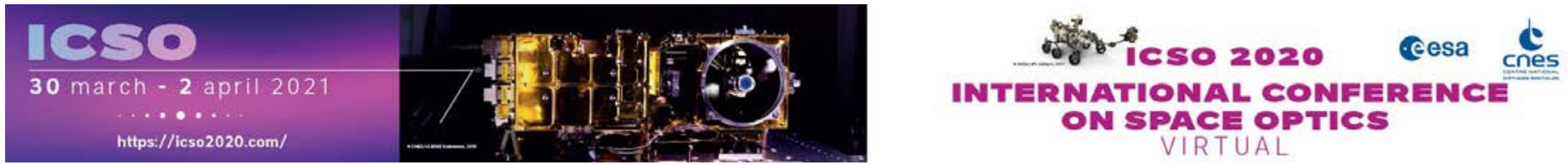

\section{Formation flying performances simulator for the shadow position sensors of the ESA PROBA-3 mission}

\section{Cesa isoporecestings denes}




\title{
Formation Flying performances simulator for the Shadow Position Sensors of the ESA PROBA-3 mission
}

\author{
Capobianco Gerardo $^{\mathrm{a}}$, Amadori Francesco ${ }^{\mathrm{a}}$, Fineschi Silvano ${ }^{\mathrm{a}}$, Bemporad Alessandro ${ }^{\mathrm{a}}$, Casti \\ Marta $^{b}$, Loreggia Davide ${ }^{a}$, Noce Vladimiro ${ }^{c}$, Pancrazzi Maurizio ${ }^{a}$, Landini Federico ${ }^{a}$, Thizy \\ Cedric $^{\mathrm{d}}$, Rougeot Raphael ${ }^{\mathrm{e}}$, Galano Damien ${ }^{\mathrm{e}}$, and Versluys Jorg ${ }^{\mathrm{e}}$

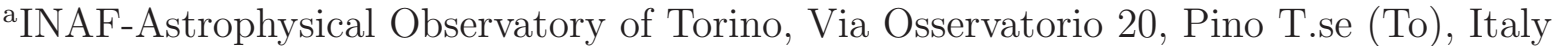 \\ b ALTEC S.p.A., Corso Marche,79, Torino, ITALY \\ ${ }^{\mathrm{c}}$ University of Florence - Dept. of Physics and Astronomy, Largo E. Fermi 2, Florence, Italy \\ ${ }^{\mathrm{d} C e n t r e}$ Spatial de Liege, Av. du Pré Aily, Liege, Belgium \\ eEuropean Space Agency-ESTEC, Keplerlaan 1, Noordwijk, The Netherlands
}

\begin{abstract}
PROBA-3 (PRoject for OnBoard Autonomy) is an ESA mission to be launched on beginning of 2023 where a spacecraft is used as an external occulter (OSC-Occulter Spacecraft), to create an artificial solar eclipse as observed by a second spacecraft, the coronagraph (CSC-Coronagraph Spacecraft). The two spacecrafts (SCs) will orbit around the Earth, with a highly elliptic orbit (HEO), with the perigee at $600 \mathrm{~km}$, the apogee at about $60530 \mathrm{~km}$ and an eccentricity of $\approx 0.81$. The orbital period is of 19.7 hours and the precise formation flight (within $1 \mathrm{~mm}$ ) will be maintained for about 6 hours over the apogee, in order to guarantee the observation of the solar corona with the required spatial resolution. The relative alignment of the two spacecrafts is obtained by combining information from several subsystems. One of the most accurate subsystems is the Shadow Position Sensors (SPS), composed of eight photo-multipliers installed around the entrance pupil of the CSC. The SPS will monitor the penumbra generated by the occulter spacecraft, whose intensity will change according to the relative position of the two satellites. A dedicated algorithm has been developed to retrieve the displacement of the spacecrafts from the measurements of the SPS. Several tests are required in order to evaluate the robustness of the algorithm and its performances/results for different possible configurations. A software simulator has been developed for this purpose. The simulator includes the possibility to generate synthetic 2-D penumbra profile maps or analyze measured profiles and run different versions of the retrieving algorithms, including the "on-board" version. In order to import the "as-built" algorithms, the software is coded using Matlab. The main aspects of the simulator, such as the results of the simulations, with the inclusion of some specific case studies, will be reported and discussed in this paper.
\end{abstract}

Keywords: PROBA-3, Space Mission, Spacecraft Formation Flying, Numerical Simulator, Solar Corona.

\section{INTRODUCTION}

In the framework of the ESA PROBA-3 mission activities [1], [2], a numerical simulator has been developed to test and validate the Shadow Position Sensors (SPS) algorithm. The PROBA-3 mission, is the fourth ESA PROBA mission, following the successful PROBA-1 (launched in 2001), PROBA-2 (2009), and PROBA-V (2013). The four PROBA missions are depicted in Figure 1.

The main objective of the PROBA-3 mission is the validation of the formation flying technologies and increase the Technology Readiness Level (TRL) of new metrology units to TRL 9 ('Actual system "flight proven" through successful mission operations' [3]). The formation flying is applied to two spacecraft, working together as a "rigid structure". One of the two spacecraft, the Coronagraph Spacecraft (CSC), host the telescope of the ASPIICS instrument [1], [2], when the second spacecraft, the Occulter Spacecraft (OSC), is working as an external occulter when the CSC is pointing the sun. Due to the large distance between the two spacecrafts, nominally

Further author information: (Send correspondence to Capobianco Gerardo)

Capobianco Gerardo: E-mail: gerardo.capobianco@inaf.it, Telephone: +39 0118101961 


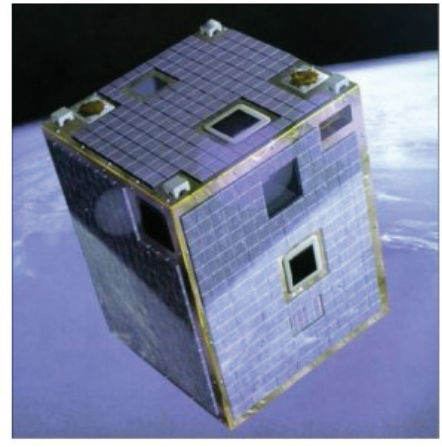

ESA PROBA-1 satellite (2001)

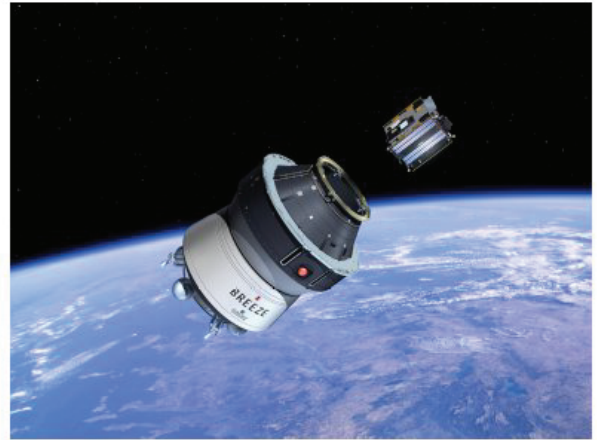

ESA PROBA-2 satellite (2009)

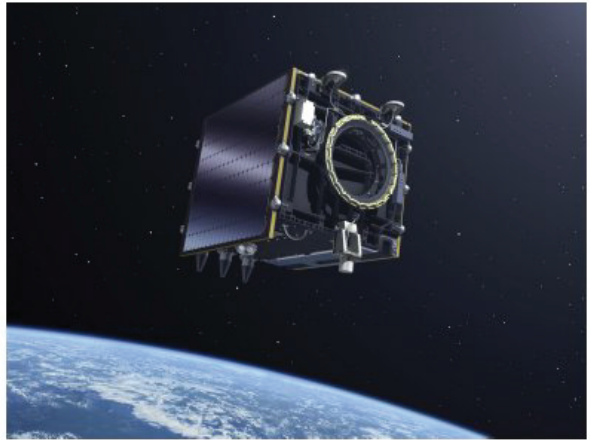

ESA PROBA-V satellite

(2013)

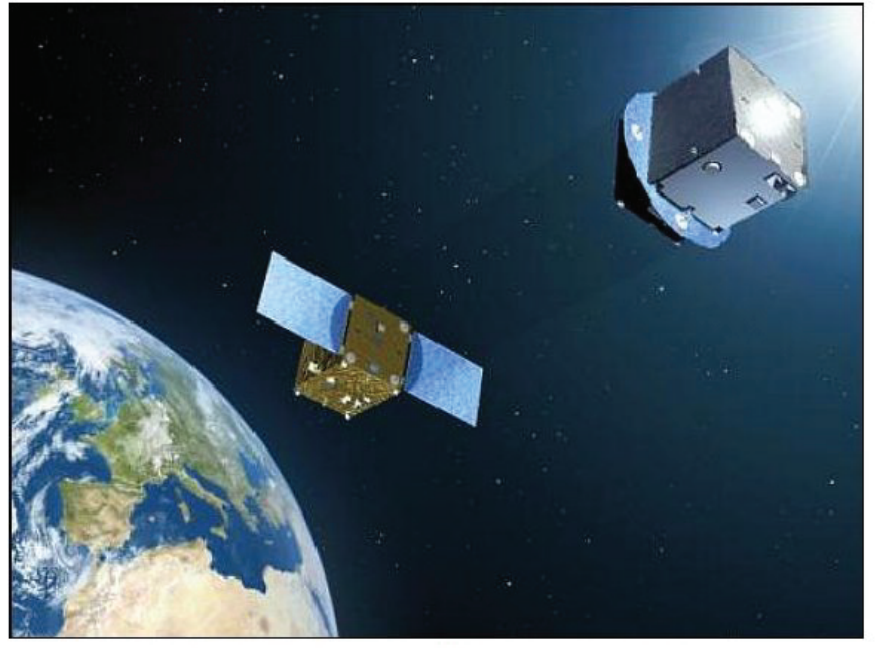

ESA PROBA-3 satellites (est. 2023)

Figure 1. The ESA PROBA satellites with the launch dates. Images credits: PROBA-1 C)Verhaert Space, ESA; PROBA-2 (CESA; PROBA-V CESA; PROBA-3 CESA.

$\approx 144 \mathrm{~m}$, the diffraction of the sunlight produced by the occulter disk will be very low, so that the solar corona should be observed in its inner region (i.e., from 1.08 solar radii). In order to achieve both the objective of the mission, technological and scientific, a sub-millimeter alignment must be guaranteed for about the $30 \%$ of the orbit period. Several subsystems are involved in the task of the metrological alignment. One of the most accurate subsystems is the SPS, acronyms of Shadow Position Sensors, a set of 8 silicon photomultipliers (SiPMs) installed around the telescope pupil (at $55 \mathrm{~mm}$ from the center of the pupil), mapping the relative displacement of the two spacecrafts by monitoring the asymmetries in the penumbra profile generated by the misalignment of the spacecrafts. A detailed review of the SPS subsystem has been recently reported in [4]. A picture of the Flight Model of the SPS board indicating the sensor number and the SPS Refernce system (SPSXF)is reported in Figure 2.

The Channel A (or nominal) is composed by the $S P S_{1}, S P S_{3}, S P S_{5}$ and $S P S_{7}$, when the channel B (or redundant) by $S P S_{2}, S P S_{4}, S P S_{6}$ and $S P S_{8}$. During the nominal operations only one channel at a time is expected to be used. The two channels will be acquired simultaneously only for calibration purposes. In agreement with the SPSXF reference frame, the $\mathrm{Y}$ and $\mathrm{Z}$ axes are referred to the lateral displacement when the $\mathrm{X}$ axis to the longitudinal. 


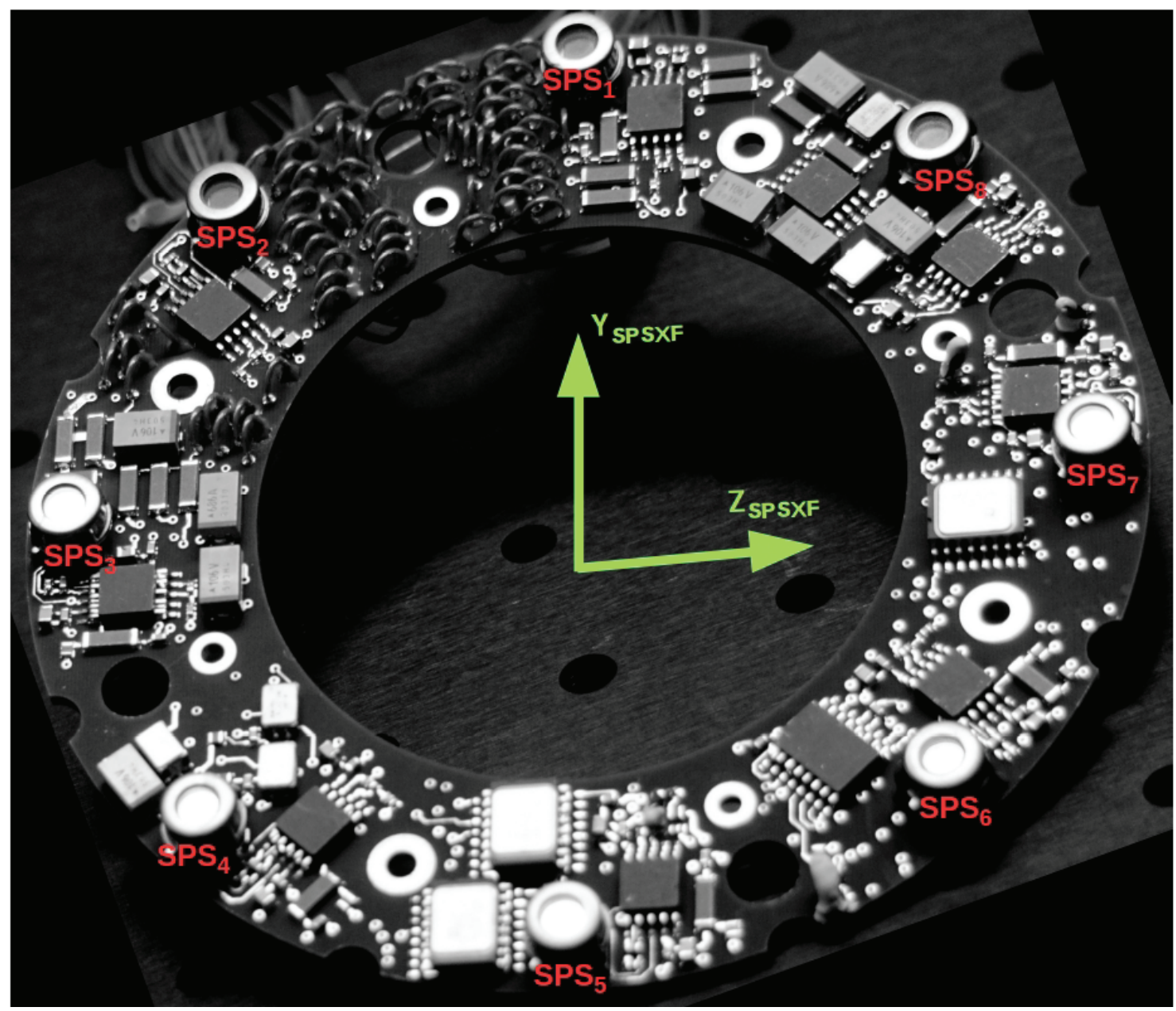

Figure 2. The SPS board (Flight Model) with the SiPMs identification number and the reference frame SPSXF.

\section{SPS ALGORITHM}

The SPS algorithm compute the relative and absolute position of the spacecrafts in order to retrieve the nominal alignment. The algorithm is described in details in [5] and is composed by five blocks, as shown in Figure 3.

The first module, the "pre-processing" module, returns the set of the SPS to be used for the computation of the spacecraft position and, based on the signal levels, select between High gain (HG) measurements or low gain (LG) when the high gain values are saturated or close to the saturation.

The second module, the "Validity Flag 1" block, returns a flag indicating the measurement validity, based on the door status (closed, open, or in transition), the SPS temperatures, and other physical parameters. This flag is used for trigger the Guidance and Navigation Control (GNC) to consider or not the positions returned by the SPS subsystem.

The third module, the "Algo Verification", returns a flag obtained by checking at the arguments of the used mathematical functions (i.e., root square and arccos, see eqn. 3) to avoid the presence of complex solutions in the position computation.

The fourth module, the "SPS Algorithm", is the core of the algorithm, responsible for computation of the spacecrafts position. Three different computational methods should be used and defined from a telecommand from ground. The first computation method is the called "Linear algorithm". The positions $Y$ and $Z$ are computed as differences between opposite SiPMs (eqn. 1 and eqn. 2). 


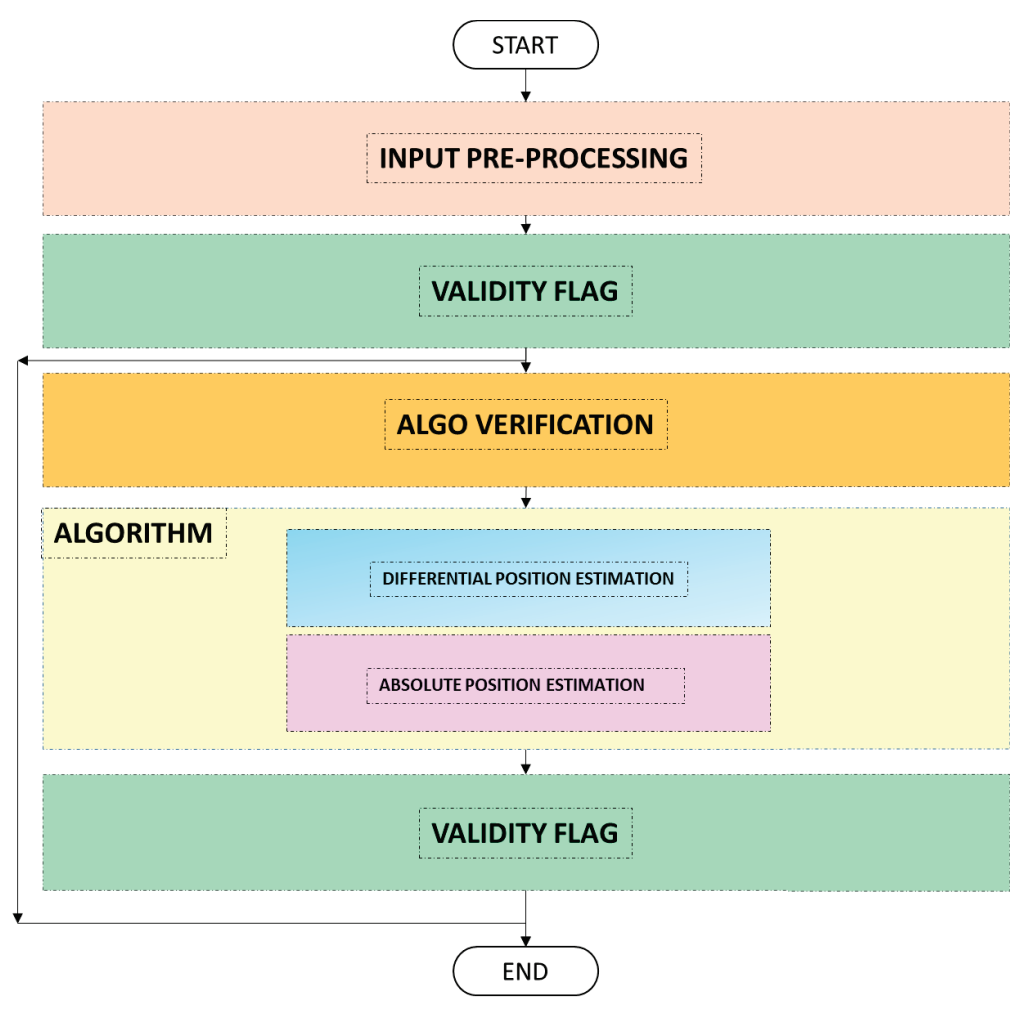

Figure 3. The SPS algorithm flow chart. See Reference [5]

$$
Y=\frac{\left(S P S_{5}-S P S_{1}\right)}{d y} ; Z=\frac{\left(S P S_{3}-S P S_{7}\right)}{d z},
$$

when the Channel A (i.e., nominal set) is used or

$$
Y=\frac{\left(S P S_{6}-S P S_{2}\right)}{d y} ; Z=\frac{\left(S P S_{4}-S P S_{8}\right)}{d z},
$$

when the Channel B (i.e., redundant set) is used. The parameters $d y$ and $d z$ are both re-configurables from ground. The second computation method is the so called "Pseudo-Paraboloid" algorithm. The positions [X, Y, Z] are computed by inverting the equations of two paraboloids fitting the penumbra intensity in two different areas. The positions are evaluated as:

$$
\left\{\begin{array}{l}
x_{0}=\frac{-K-\sqrt{K^{2}-4 H\left(L-D_{\text {SPSpos }}\right)}}{2 H}+J d_{0}^{2} \\
y_{0}=2 \sqrt{-\frac{A}{3}} \cdot \cos \left\{\frac{1}{3}\left[\cos ^{-1}\left(\frac{\left(I_{5}-I_{1}\right) c^{3}}{4 \sqrt{-\left(\frac{A}{3}\right)^{3}}}\right)+4 \pi\right]\right\} \\
z_{0}=2 \sqrt{-\frac{A}{3}} \cdot \cos \left\{\frac{1}{3}\left[\cos ^{-1}\left(\frac{\left(I_{3}-I_{7}\right) c^{3}}{4 \sqrt{-\left(\frac{A}{3}\right)^{3}}}\right)+4 \pi\right]\right\}
\end{array}\right.
$$

where $A$ is given by (4):

$$
A=c^{3}\left(\frac{1}{a}+\frac{2 r_{S P S}}{b^{2}}+\frac{3 r_{S P S}^{3}}{c^{3}}\right)
$$

$a, b, c, H, K, J$ and $L$ are the parameters of the $3 \mathrm{D}$ pseudo-paraboloid (re-configurable from ground), $r_{S P S}$ is the position where the SPS are installed w.r.t. the center of the telescope pupil (i.e, 55mm), $D_{S P S p o s}$ is the 
average of the differences between the measured values and the ones referred to the aligned configuration and $d_{0}=\sqrt{y_{0}^{2}+z_{0}^{2}}$.

The parameters of the pseudo-paraboloid (i.e, $a, b, c, H, K, J$ and $L$ ) are theoretically estimated before the launch, but needs to be calibrated in-flight [6]. The third computation method is providing the average of the results of the two described methods.

The last module, called "Validity Flag 2", compares the ongoing calculated positions with the previous one, estimating the lateral and longitudinal speeds and checking their compatibility with the expected ones. It returns two flags related to each set of SPSs.

\section{END-TO-END SIMULATOR}

The end-to-end simulator has been developed in Matlab ${ }^{\circledR}$ since the SPS algorithm is coded using this language. The main purpose of the simulator is to provide, to the user, a tool for testing the complete SPS measurement chain, test the robustness and performances of the algorithm, verify how the errors on the XYZ coordinates returned by the algorithm changes while changing the penumbra fitting parameters. Moreover, the simulator will give a support for the activities during the commissioning phase, evaluating possible critical conditions related to the retrieved fitting parameters. In Figure 4 is shown the main page of the simulator.

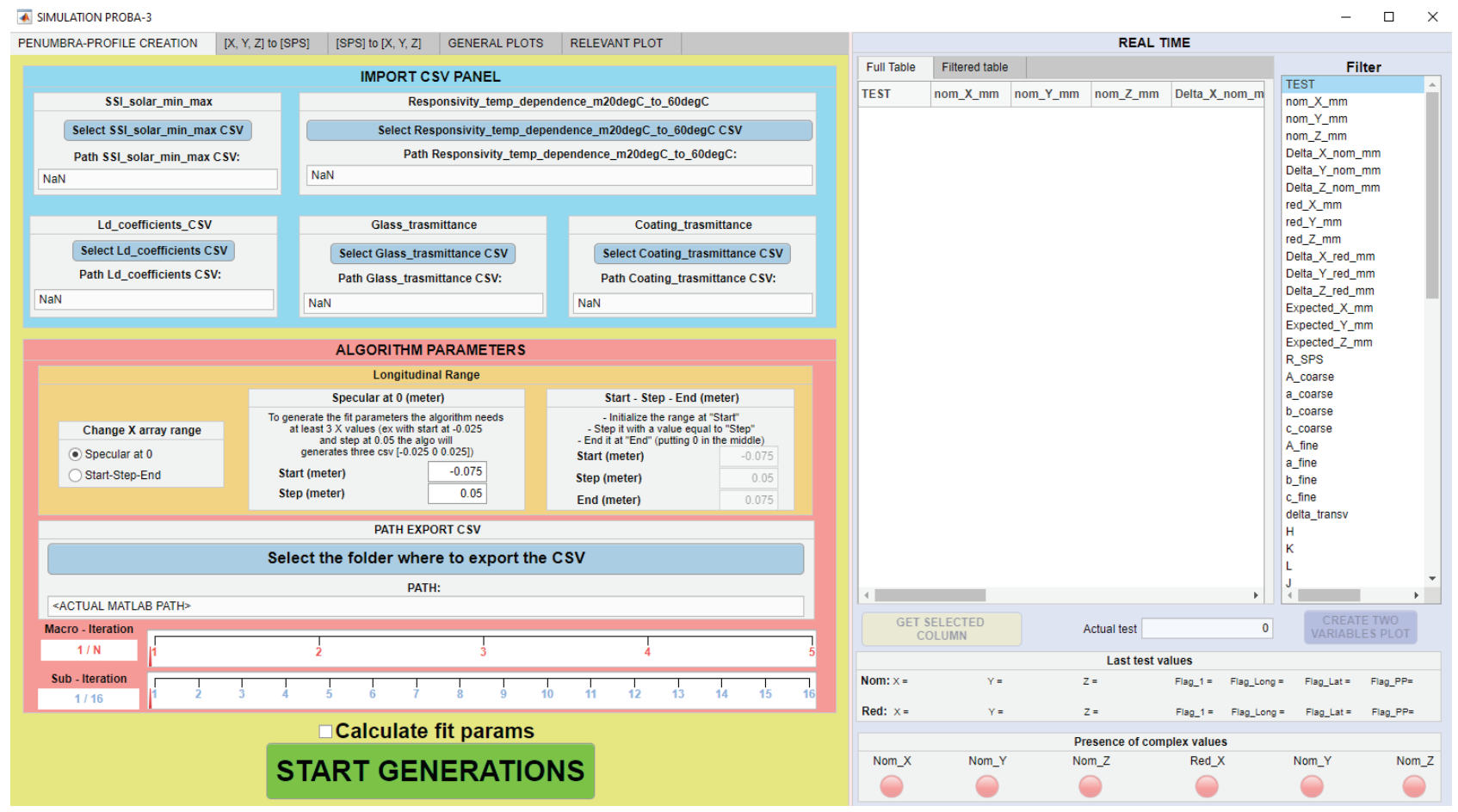

Figure 4. The main page of the simulator

The tool allows the user to simulate different configurations, by changing all the re-configurable parameters of the algorithm and/or changing the measurements from the sensors (i.e., temperatures, door status, DNs generated by the SiPMs). The simulator can also generate synthetic penumbra profiles maps according to the geometry of the flight formation [ 7]. From a given penumbra profile map and spacecraft position, the simulator is also able to determine the irradiances detected by each SiPM and the DNs generated.

As shown in the Figure 4, on the top-left side of the simulator there are five macro sections (tabs), three related to numerical computation functionalities and two for results preview. In the next section, the numerical computation functionalities will be detailed. 


\subsection{2-D synthetic penumbra profile maps generation}

Through the interface shown in Figure 5 ("Penumbra Profile Creation" tab), the user can generate a 2-D synthetic penumbra map.

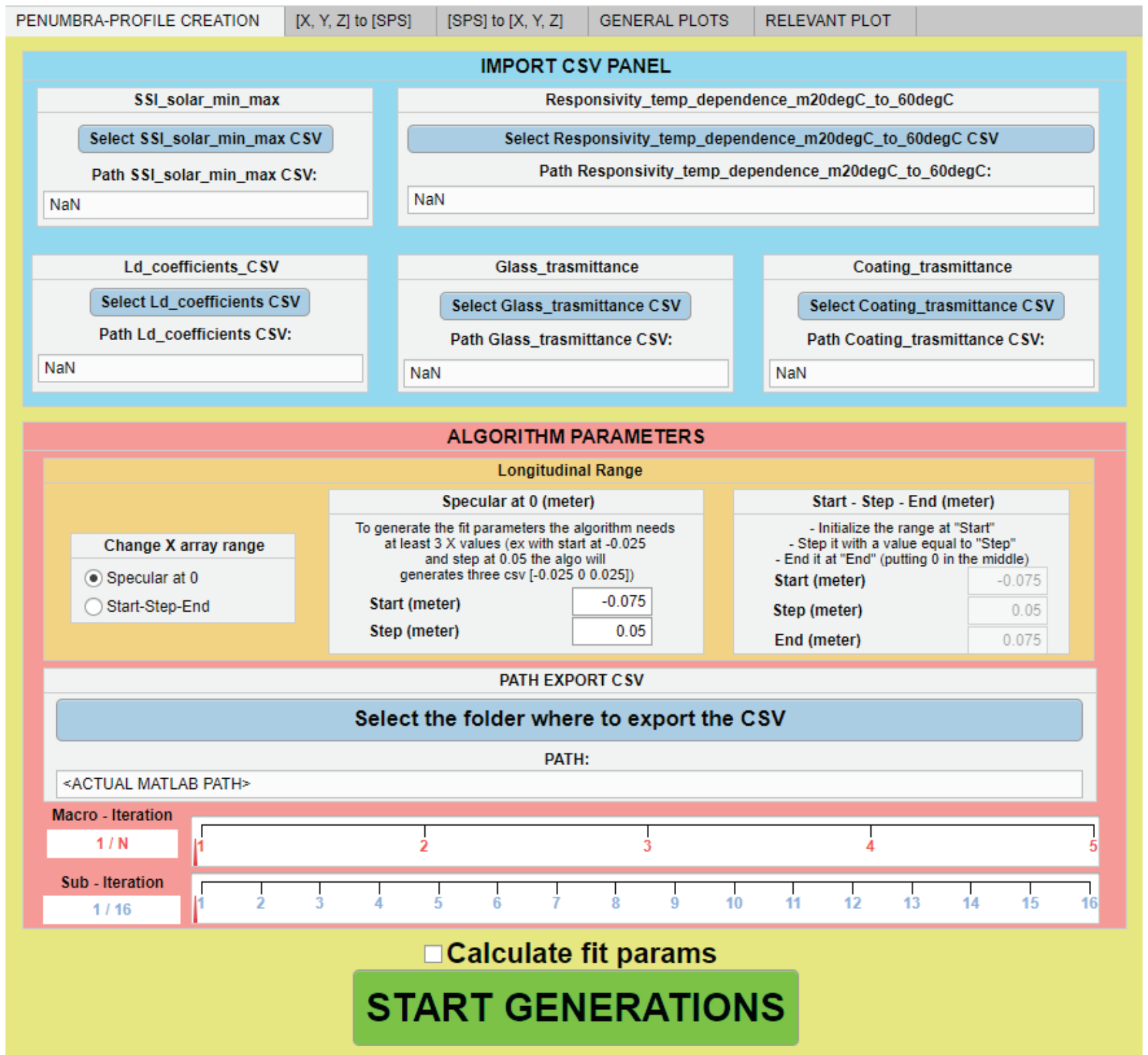

Figure 5. Penumbra profile generation tab.

The inputs for the algorithm are:

- "SSI_solar_min_max": a .CSV file reporting the solar irradiance related to the sun activity period (maximum or minimum);

- "Responsivity_temp_dependence_m20degC_to_60degC": a .CSV file containing the SiPMs responivities at different temperatures;

- "Ld_coefficients_CSV": a .CSV file with the solar limb darkening coefficients; 
- "Glass_transmittance": a .CSV file containing the transmissivity data of the windows in front of the SiPMs;

- "Coating_transmittance": a CSV file with the data related to the coatings of the SiPMs;

- "Longitudinal Range": the longitudinal distances (X) where evaluate the penumbra irradiance on the lateral plane (Y-Z). The values of the penumbra irradiance is the average of the irradiances at the selected distances $(\mathrm{X})$. The distances can be selected manually by using the "Start-Step-End" option, where the user will introduce the first and last longitudinal distances (i.e., start point and end point) and the step size or simmetrically to the nominal position by using the "Specular to 0" option. In this case only the start point and the step size are required;

- "Path Export CSV", the path where penumbra profile map and the fitting parameters will be exported.

The 2-D penumbra maps generated covers the lateral range (Y-Z) from $[-155,-155] \mathrm{mm}$ to $[+155,+155] \mathrm{mm}$ with a resolution of $0.5 \mathrm{~mm}$.

\subsection{Simulation of the expected output from the SPS}

This interface, depicted in Figure 6 ("[X,Y,Z] to [SPS]" tab), allows the user to evaluate the expected output from the SPS in Digital Units (DN) starting from a penumbra profile map.

The inputs for the algorithm are:

- "COORDINATES": the relative position of the spacecrafts $[\mathrm{X}, \mathrm{Y}, \mathrm{Z}]$ to simulate;

- "SPS THETA": the angle of each SiPM in radians (the 0 rad angle is aligned with the $Z_{S P S X F}$ );

- "CSV PATH": the location of the files containing the penumbra profile information and the fitting parameters.

The algorithm will display the currents and the DNs generated by each SIPMs in the current configuration. These values can be exported in files. A graphical visualization of the SPS disposition compared to the centred position and the OSC umbra is also displayed.

\subsection{Spacecrafts Position Retrieval}

This section is dedicate to run the SPS algorithm. From the output of the SiPMs in DN and by setting the re-configurable parameters, the position of the flight formation is returned. The tab is shown in Figure 7 .

The main settings are:

- "Raw Data": Containing the readings from the sensors (i.e., High Gain and Low Gain DNs from the SiPMs, Temperatures, Door status,...);

- "Reconf Params": all the re-configurable parameters of the algorithms (i.e., " $K_{i}$ ", "pp_coarse", "pp_fine", "linear", "long", "thresholds", "algorithm" and "radius SPS"). The re-configurable parameters are listed below;

- "X, Y, Z Expected": the expected position of the formation. This values are used for evaluate the errors in the algorithm retrieved position.

The lower part of the page contains the settings for the test to be performed. The user can select the number of test to be performed, the parameters to change and their variation range (absolute or relative), to change randomly more than one parameter by performing a Montecarlo simulation, etc. The results can be exported in .csv files and/or displayed in real-time mode.

The re-configurable parameters are the same expected for the "flight" version of the algorithm: 


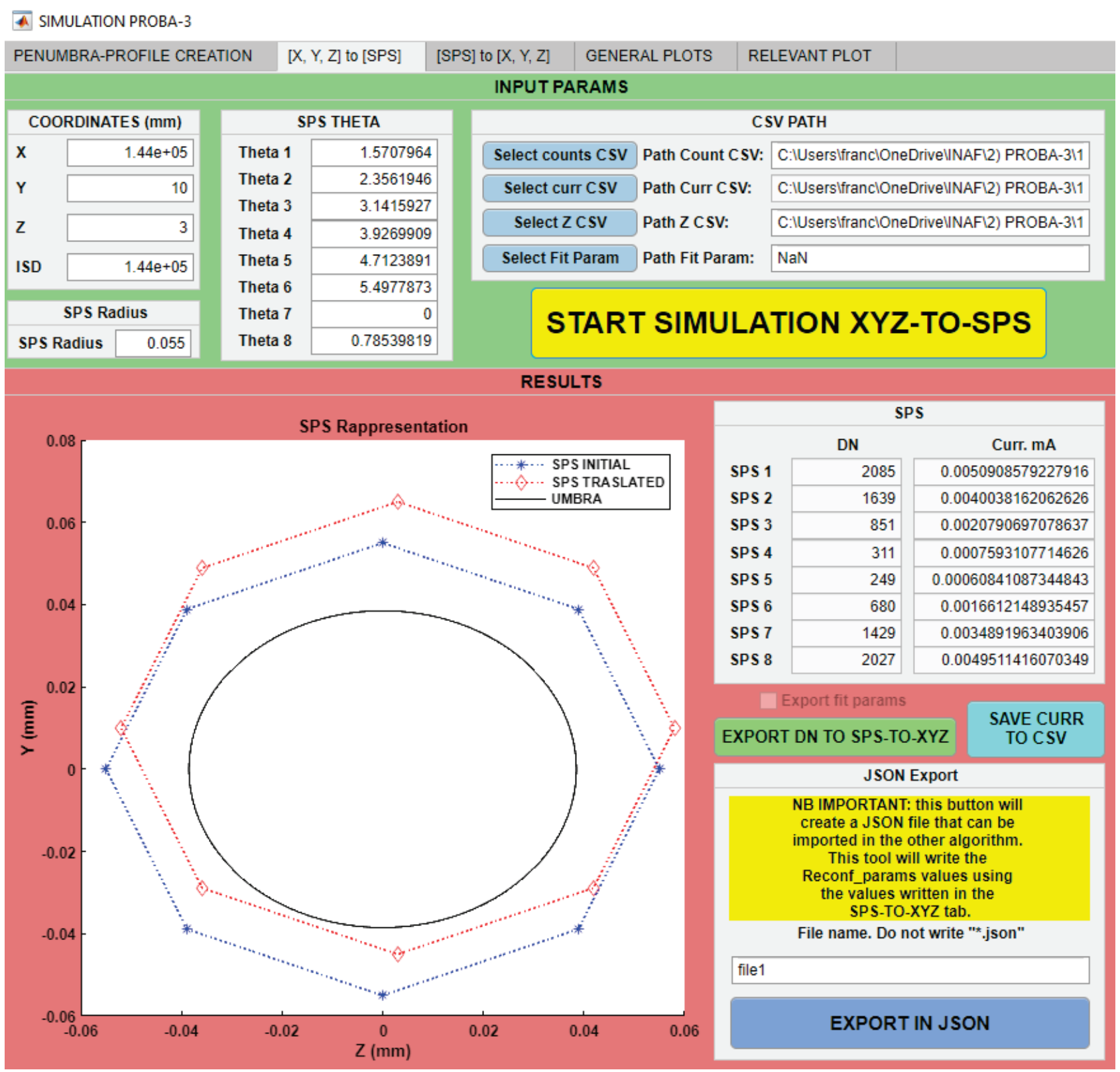

Figure 6. Simulation of the SPS readings tab.

- the $K_{i}$ parameters, 8 parameters taking into account the variation in terms of responsivity of the SiPMs during the mission life. These parameters works also as a cross-calibration parameter between the 8 SiPMs;

- pp_coarse parameters, are the four parameters (i.e., a, b, c and A) fitting the pseudo-paraboloid in a large region (see equation 3);

- pp_fine parameters, are the four parameters (i.e., a, b, c and A) fitting the pseudo-paraboloid in a small region around the "aligned" configuration. The "delta_transv" parameter define the limit of this region;

- long, are the five parameters (i.e., H, K, L, J and delta_R)for the evaluation of the longitudinal position $(\mathrm{X})$, accordingly to equation 3 ; 


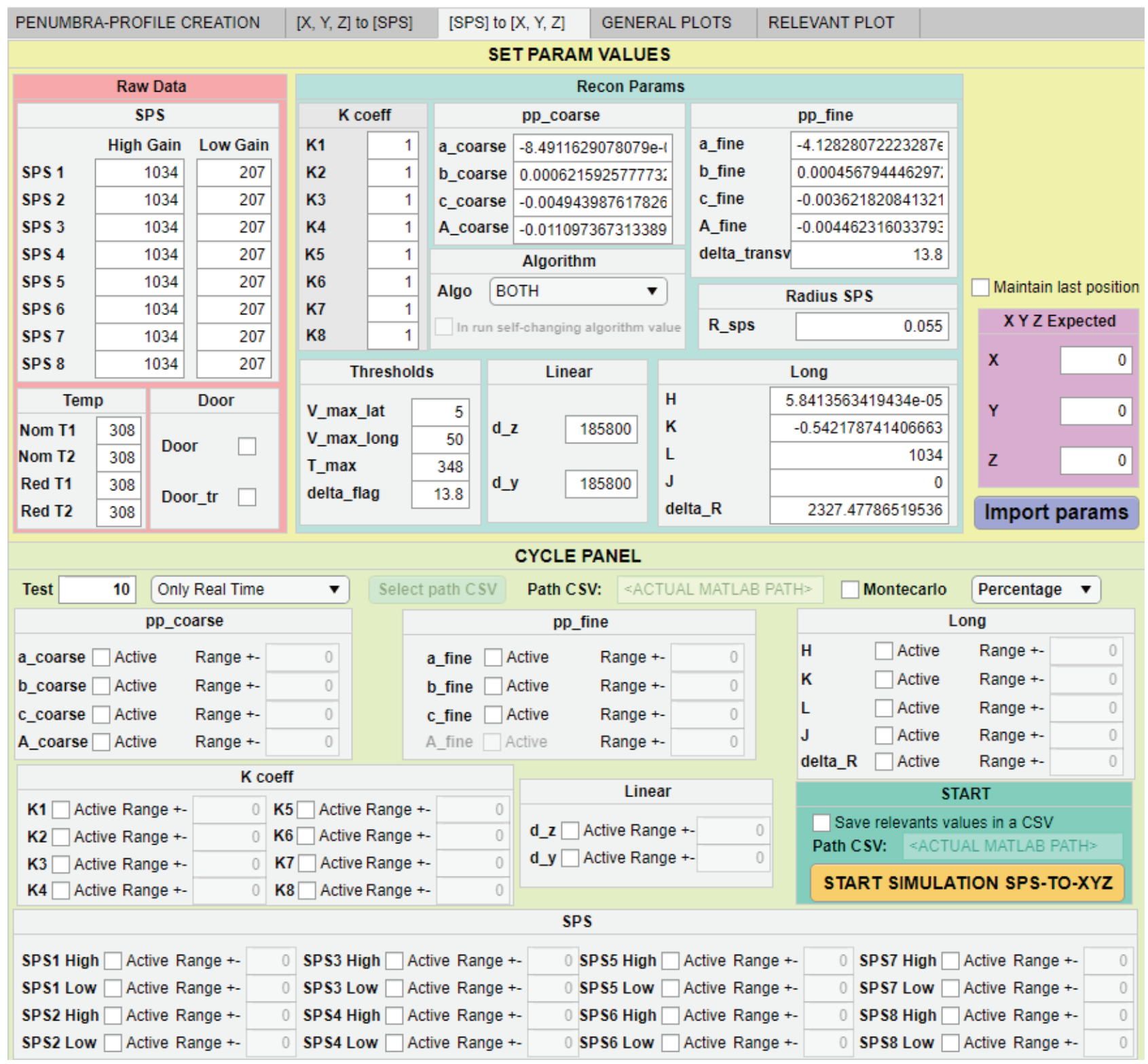

Figure 7. The tab for run the SPS algorithm.

- linear, contains the two parameters for the evaluation of (Y and Z) by using the "linear" algorithm, $d_{y}$ and $d_{z}$, see equations 1 and 2 ;

- thresholds, are the limit values for the activation of the flags;

- algorithm, allows the user to select if use the "linear" algorithm, the "pseudo-paraboloid" algorithm or both;

- radius SPS is the distance of the SiPMs w.r.t. the center of the ASPIICS pupil (i.e., $55 \mathrm{~mm}$ ).

\subsection{Results display and real-time computational output}

The results of the simulations are shown in real-time in a table on the right panel (see Figure 8). The plots showing the results for the three axis [X,Y and Z] and a user defined 2 variables plot are shown in two dedicated 
tabs(see Figure 9).

\begin{tabular}{|c|c|c|c|c|c|}
\hline \multicolumn{6}{|c|}{ REAL TIME } \\
\hline Full Table & Filtered table & & & & Filter \\
\hline TEST & nom_X_mm & nom_Y_mm & nom_Z_mm & Delta_X_nom & $\begin{array}{l}\text { TEST } \\
\text { nom_X_mm }\end{array}$ \\
\hline 0 & $-2.2060 \mathrm{e}+03$ & $-5.9841 e-15$ & $-5.9841 e-15$ & $-2.20 \wedge$ & nom_Y_mm \\
\hline 1 & $-2.1923 e+03$ & $-5.9930 \mathrm{e}-15$ & $-5.9930 \mathrm{e}-15$ & -2.19 & nom_Z_mm \\
\hline 2 & $-2.1786 e+03$ & $-6.0019 e-15$ & $-6.0019 \mathrm{e}-15$ & -2.17 & Delta_X_nom_mm \\
\hline 3 & $-2.1648 \mathrm{e}+03$ & $-6.0107 e-15$ & $-6.0107 e-15$ & -2.16 & $\begin{array}{l}\text { Delta_Y_nom_mm } \\
\text { Delta_Z_nom_mm }\end{array}$ \\
\hline 4 & $-2.1511 e+03$ & $-6.0195 \mathrm{e}-15$ & $-6.0195 e-15$ & -2.15 & red_X_mm \\
\hline 5 & $-2.1374 \mathrm{e}+03$ & $-6.0283 e-15$ & $-6.0283 e-15$ & -2.13 & red_Y_mm \\
\hline 6 & $-2.1236 \mathrm{e}+03$ & $-6.0371 e-15$ & $-6.0371 e-15$ & -2.12 & red_Z_mm \\
\hline 7 & $-2.1099 \mathrm{e}+03$ & $-6.0458 \mathrm{e}-15$ & $-6.0458 \mathrm{e}-15$ & -2.10 & $\begin{array}{l}\text { Delta_X_red_mm } \\
\text { Delta_Y_red_mm }\end{array}$ \\
\hline 8 & $-2.0961 e+03$ & $-6.0545 e-15$ & $-6.0545 e-15$ & -2.09 & Delta_Z_red_mm \\
\hline 9 & $-2.0823 e+03$ & $-6.0632 e-15$ & $-6.0632 e-15$ & -2.08 & Expected_X_mm \\
\hline 10 & $-2.0685 e+03$ & $-6.0719 \mathrm{e}-15$ & $-6.0719 \mathrm{e}-15$ & -2.06 & Expected_Y_mm \\
\hline 11 & $-2.0547 e+03$ & $-6.0805 e-15$ & $-6.0805 e-15$ & -2.05 & Expected_Z_mm \\
\hline 12 & $-2.0410 e+03$ & $-6.0891 e-15$ & $-6.0891 \mathrm{e}-15$ & -2.04 & $\begin{array}{l}\text { R_SPS } \\
\text { A_coarse }\end{array}$ \\
\hline 13 & $-2.0271 e+03$ & $-6.0977 \mathrm{e}-15$ & $-6.0977 e-15$ & -2.02 & a_coarse \\
\hline 14 & $-2.0133 e+03$ & $-6.1062 \mathrm{e}-15$ & $-6.1062 e-15$ & -2.01 & b_coarse \\
\hline 15 & $-1.9995 e+03$ & $-6.1148 \mathrm{e}-15$ & $-6.1148 \mathrm{e}-15$ & -1.99 & c_coarse \\
\hline 16 & $-1.9857 e+03$ & $-6.1233 \mathrm{e}-15$ & $-6.1233 e-15$ & -1.98 & A_fine \\
\hline 17 & $-1.9718 e+03$ & $-6.1318 \mathrm{e}-15$ & $-6.1318 \mathrm{e}-15$ & -1.97 & b_fine \\
\hline 18 & $-1.9580 e+03$ & $-6.1402 \mathrm{e}-15$ & $-6.1402 \mathrm{e}-15$ & -1.95 & c_fine \\
\hline 19 & $-1.9441 e+03$ & $-6.1487 e-15$ & $-6.1487 e-15$ & -1.94 & delta_transv \\
\hline 20 & $-1.9303 e+03$ & $-6.1571 \mathrm{e}-15$ & $-6.1571 \mathrm{e}-15$ & -1.93 & $\begin{array}{l}\mathrm{H} \\
\mathrm{K}\end{array}$ \\
\hline 21 & $-1.9164 e+03$ & $-6.1655 \mathrm{e}-15$ & $-6.1655 \mathrm{e}-15$ & -1.911 & L \\
\hline 22 & $-1.9025 \mathrm{e}+03$ & $-6.1738 \mathrm{e}-15$ & $-6.1738 \mathrm{e}-15$ & $-1.90 \cdot$ & $\mathrm{J}$ \\
\hline & & & & ' & 4 \\
\hline $\begin{array}{r}\text { GET S } \\
\text { CO }\end{array}$ & $\begin{array}{l}\text { ELECTED } \\
\text { LLUMN }\end{array}$ & & ctual test & 300 & $\begin{array}{c}\text { CREATE TWO } \\
\text { VARIABLES PLOT }\end{array}$ \\
\hline & & & Last test valc & ues & \\
\hline Nom: $x=374$ & $Y=$ & $-7.8715308 \mathrm{e} \ldots \quad z=$ & $=-7.8715308 \mathrm{e} \ldots$ & Flag_1 $=1 \quad$ Flag_Long $=0$ & Flag_Lat $=1 \quad$ Flag_PP $=1$ \\
\hline Red: $x=374$ & 4.5248 & $-1.1132026 \mathrm{e}-\ldots \mathrm{z}=$ & $=0$ & Flag_Long $=0$ & Flag_Lat $=1 \quad F l a g_{-} P P=1$ \\
\hline & & Pres & sence of compl & lex values & \\
\hline Nom_X & Nom_Y & Nom & & Red_X & Nom_Y \\
\hline & & & & & \\
\hline
\end{tabular}

Figure 8. The tab where the simulation results are shown in real-time.

A detailed description of the simulator, including the Matlab ${ }^{\circledR}$ source code is reported in [ 8].

\section{SIMULATOR VALIDATION}

The simulator is expected to be validated in all the produced output.

- The "penumbra profile generation" module validation is in progress by using the full Sun disk data from the Solar Dynamics Observatory mission (SDO).

- The "expected output from the SiPMs" module has been validated by checking the conversion between irradiances and DNs generated by the SiPMs with the electronics design [ 9] .

- The "spacecrafts Position Retrieval" has been validated by checking the results (i.e., X,Y and Z) provided by the simulator for known S/C positions. By the way, during the debugging of the simulator, the validation of the results has been performed by checking the results of the simulator with the results given by running the SPS algorithm stand-alone.

\section{APPLICATION OF THE SIMULATOR TO THE SPS ALGORITHM TEST}

The simulator has been used for the SPS algorithm debugging and is currently largely used for the SPS algorithm performances test. The main questions addressing this study are: 


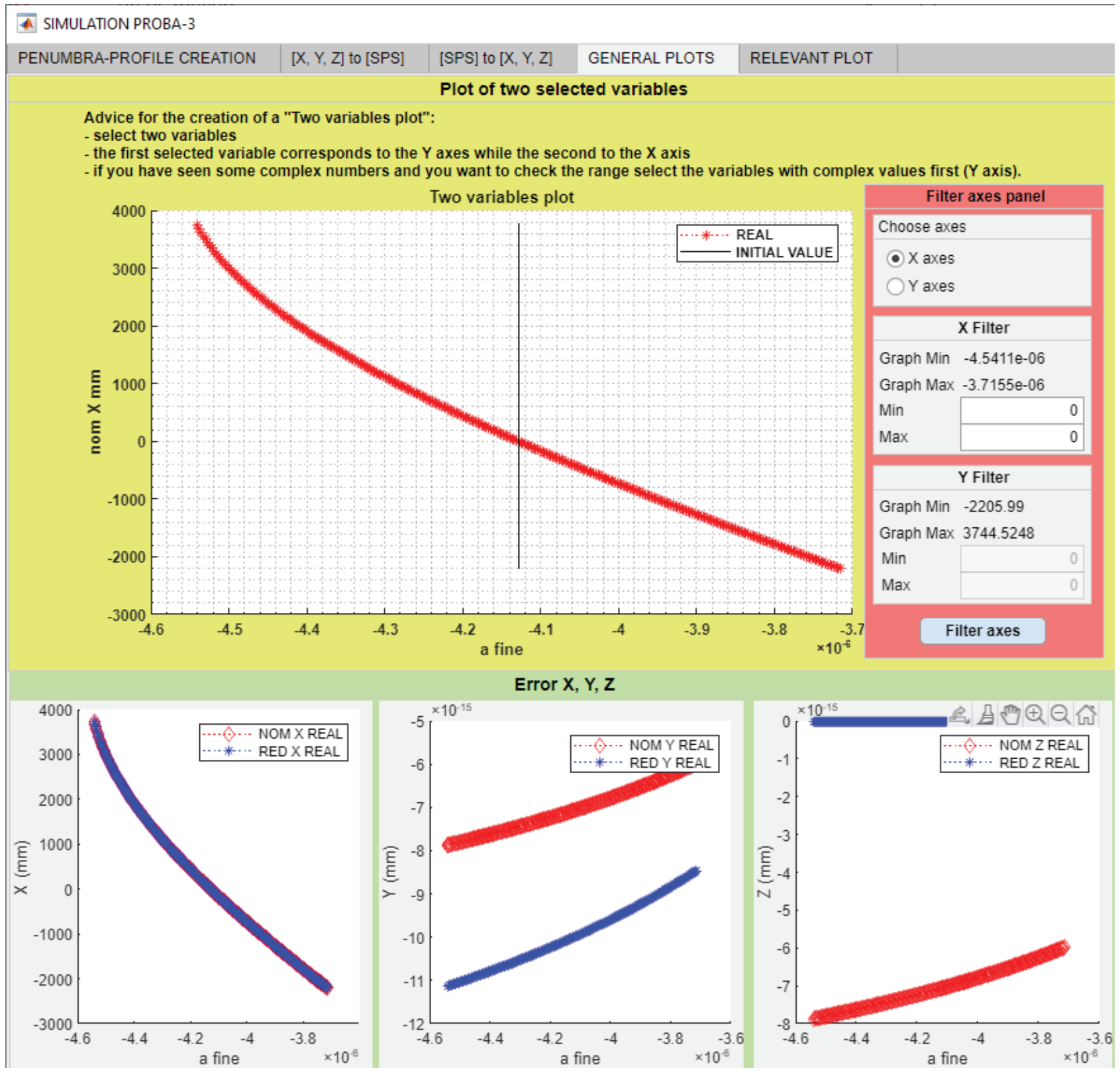

Figure 9. The tab dedicated to the data display (graphs).

- How much the algorithm is sensitive to the penumbra profile knowledge?

- How much the algorithm is sensitive to measurement errors?

- Are the SPS algorithm results still valid in some "exotic" S/Cs configurations?

\subsection{SPS algorithm sensitivity to the penumbra profile knowledge}

This study was performed in three steps. Starting from several S/C alignment configurations and using the nominal parameters for the fit of the penumbra profile, we checked the results returned by the SPS algorithm. The second step was to change for these S/C configurations a single fitting parameter at a time, finding for each parameter a range of variability where the results of the algorithms are still mathematically/physically correct 
(i.e., no complex values). The last step was, starting from the information coming from the first two steps, (i.e., expected results and maximum variation range of the parameters) to apply a "Montecarlo" simulation, where all the parameters are changing randomly inside a fixed range. The results shows that the SPS algorithm returns a position with the required accuracy, when the penumbra parameters is fitted with a precision of $\pm 1 \%$ in the full range or $\pm 2 \%$ inside the "fine" range. In Figure 10 the results of the Montecarlo simulation at the nominal inter-satellite distance (i.e., $\mathrm{X}=0$ ) and for a lateral misalignment of $\mathrm{Y}=+10 \mathrm{~mm}$ and $\mathrm{Z}=+10 \mathrm{~mm}$ is shown. The variation of the parameters was set to $\pm 2 \%$ and $10^{5}$ runs were performed.
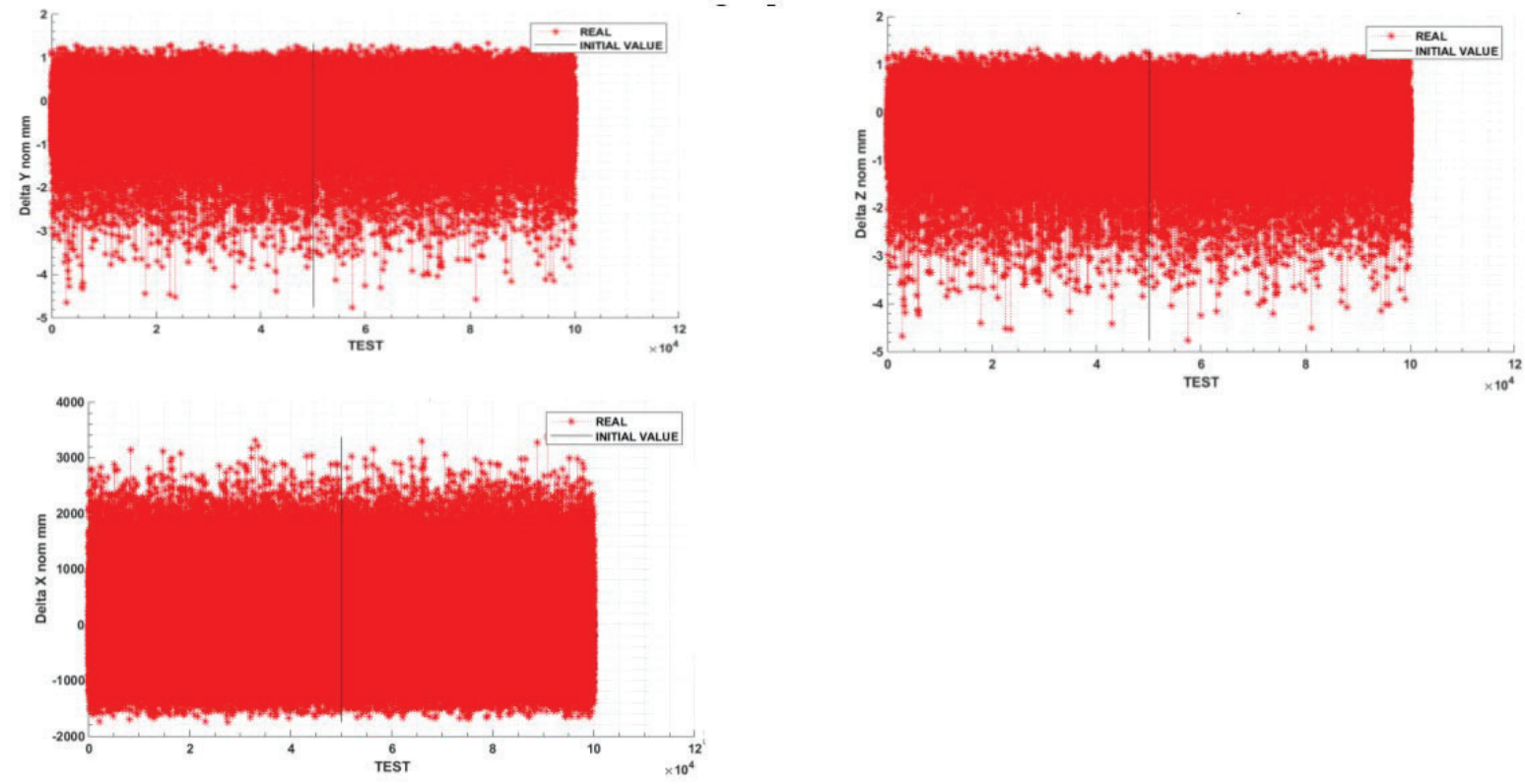

Figure 10. The Montecarlo simulation for the position $[0,10,10]$ with a variation range of the parameters of $\pm 2 \%$ and $10^{5}$ runs. Deviations from expected results for $\mathrm{Y}$ axis (top left), Z axis (top right) and $\mathrm{X}$ axis (bottom).

\subsection{SPS algorithm sensitivity to measurement errors}

Since the SPS algorithm is based on differences between the readings of opposite SiPMs (see section 2), this test evaluates the errors in the retrieved position introducing asymmetries in the measurements. The results are in agreement with what expected from the electronics design [9] and they shows as depicted in Figure 11 a variation of about 46 micron/DN. This information is reported as accuracy in radiometric calibration of the SiPMs that should be better than $1 \%$.

\subsection{Specific test cases}

Several configurations have been tested by using the simulator. The results provided useful information about the limitations of algorithm to retrieve specific configurations (i.e., one SiPM measure 0 DN, 2 SiPMs in the same side of the shadow, one SiPM saturated, and so on...).

The simulator has been also used as a tool for investigate the number of iteractions required for the algorithm to return positions with the required accuracy, (usually $<4$ ). A different application of the simulator has been the evaluation of the computation method (linear, pseudo-paraboloid, or the average of the two) providing better performances for specific $\mathrm{S} / \mathrm{C}$ configurations. The map shown in Figure 12 , referred to $\mathrm{X}=0, \mathrm{X}=100$ and $\mathrm{X}=500 \mathrm{~mm}$ ( $\mathrm{X}=0$ correspond to the nominal inter-satellite distance) depict on the lateral plane, the algorithm giving higher accuracy.

The last application we used the simulator is for the check of the region where evaluate the pseudo-paraboloid fitting parameters. 


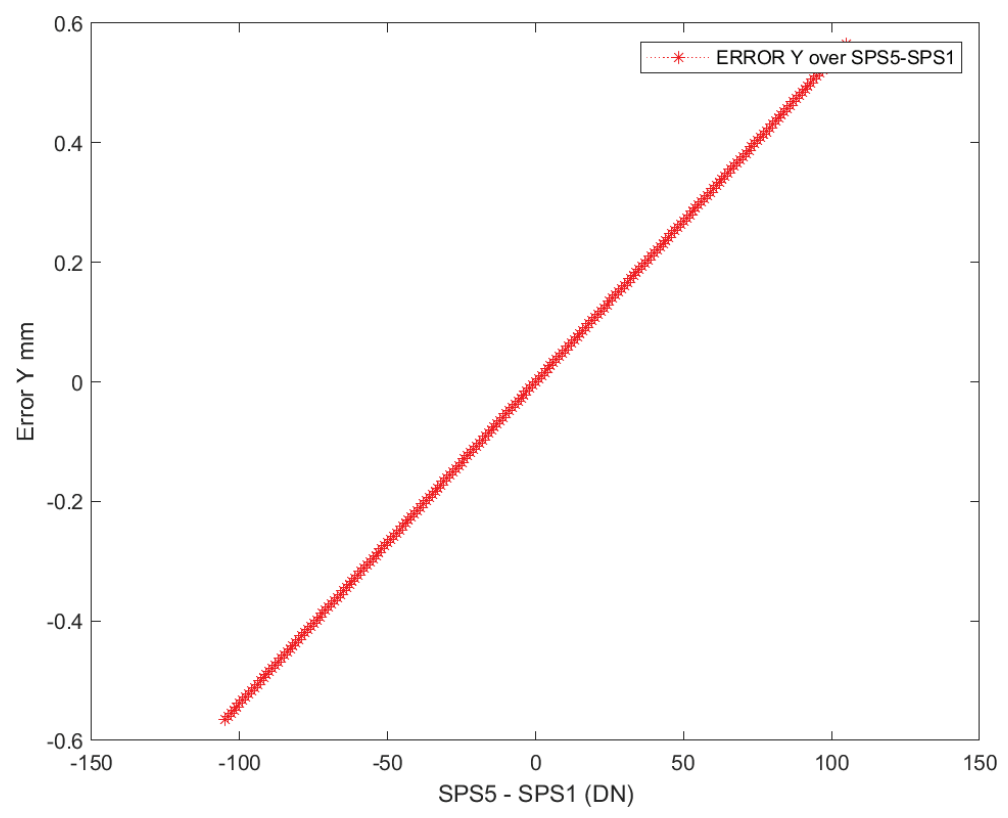

Figure 11. Error on the Y position as function of the difference between the measurements provided by $S P S_{5}$ and $S P S_{1}$.

\section{CONCLUSIONS}

In this work, we shown and described the numerical simulator developed for debugging, verify and characterize the performances of the algorithm developed for the SPS subsystem of the ESA/PROBA-3 mission. The validation of the simulator itself has been reported, as some specific results of the verification campaign of the SPS algorithm. The use of such a simulator should be useful also during the commissioning and nominal phase of the mission as supporting tool for the calibration activities.

\section{ACKNOWLEDGMENTS}

The authors acknowledge the support provided by the PROBA-3 Managerial and Technical Staff of the European Space Agency (ESA) within the contract with CSL (Centre Spatial de Liège) and subcontractors, subscribed for the Payload Instrument design and development (C/D Phases).

F. Amadori acknowledges professor Dr. Levi Giuseppe (University of Bologna) for his teachings and his ability to instill his passion and dedication to Astronomy research fields. 

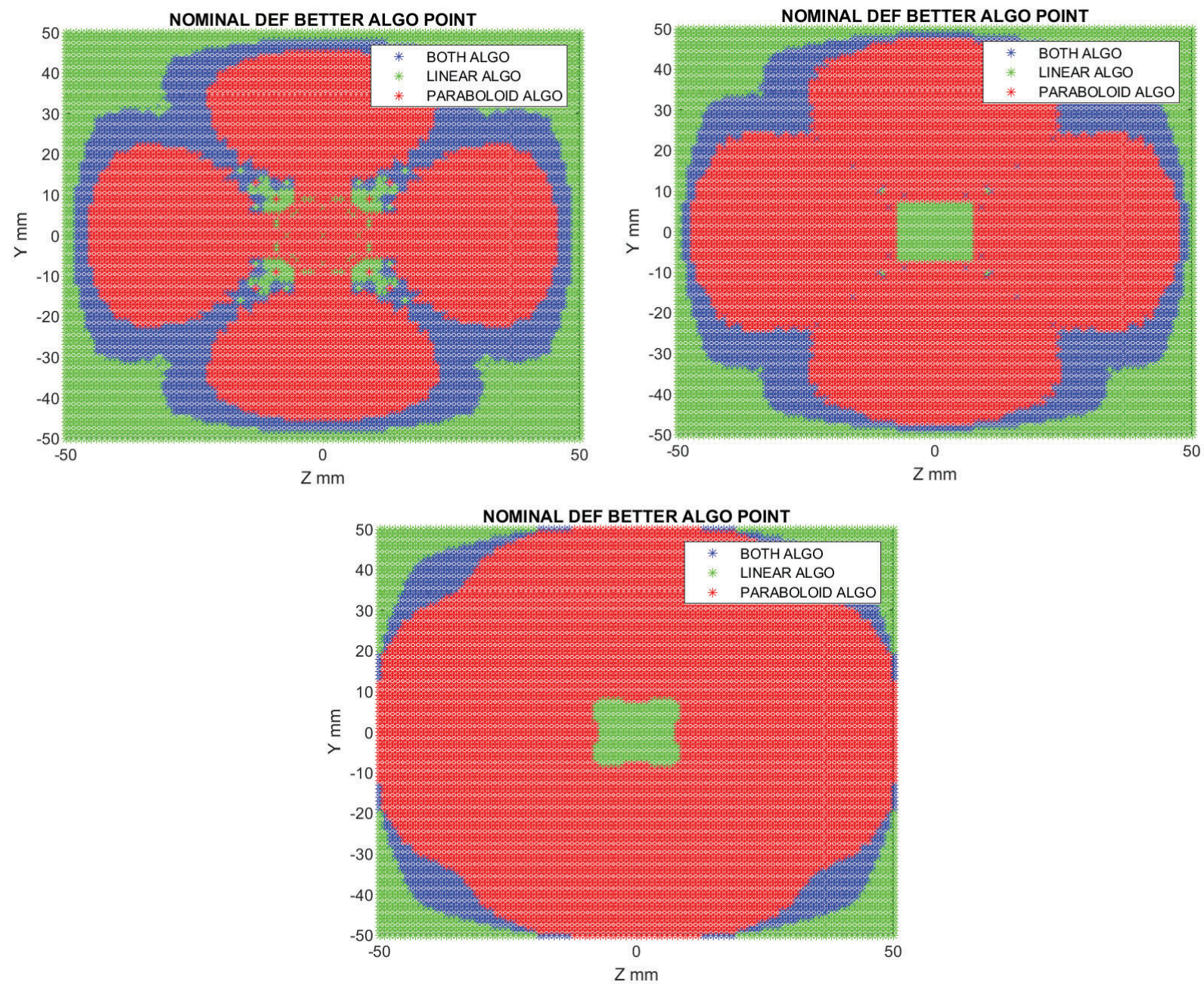

Figure 12. Identification of the algorithm giving better performances on the lateral plane (Y-Z) for inter-satellite distance nominal (top left), nominal+100 mm (top right) and nominal+500 mm (bottom)

\section{REFERENCES}

[1] Lamy, P., Dame, L., Vives, S., and Zhukov, A., "ASPIICS: a giant coronagraph for the ESA/PROBA-3 Formation Flying Mission," in [Space Telescopes and Instrumentation 2010: Optical, Infrared, and Millimeter Wave], Proc. SPIE 7731 (2010). https://doi.org/10.1117/12.858247.

[2] Galano, D. and et al., "Development of ASPIICS: a coronagraph based on Proba-3 formation flying mission," in [Space Telescopes and Instrumentation 2018: Optical, Infrared, and Millimeter Wave], Proc. SPIE 10698 (2018). https://doi.org/10.1117/12.2312493.

[3] Mankins, J. C., [TECHNOLOGY READINESS LEVELS: A White Paper], NASA (1995).

[4] Noce, V., Loreggia, D., Capobianco, G., Fineschi, S., Bemporad, A., Casti, M., Buckley, S., Romoli, M., Focardi, M., Belluso, M., Thizy, C., Hermans, A., Galano, D., and Versluys, J., "Metrology on-board PROBA-3: The shadow position sensors subsystem," Advances in Space Research (2020). https://doi.org/10.1016/j.asr.2020.08.004.

[5] Casti, M., Bemporad, A., Fineschi, S., Capobianco, G., Loreggia, D., Noce, V., Landini, F., Thizy, C., and Damien Galano, R. R., "PROBA-3 formation-flying metrology: algorithms for the shadow position sensor system," in [International Conference on Space Optics - ICSO 2018], Proc. SPIE 11180, 1118082 (2019). https://doi.org/10.1117/12.2536209. 
[6] Capobianco, G., Fineschi, S., Loreggia, D., Bemporad, A., Landini, F., Casti, M., Noce, V., Romoli, M., Galano, D., and Thizy, C., "The in-flight calibration procedures of the Shadow Positioning Sensors (SPS) a very accurate optical metrology system of the ESA-PROBA3 formation flying mission," in [2019 IEEE 5th International Workshop on Metrology for AeroSpace (MetroAeroSpace)], 479-483 (2019).

[7] Bemporad, A., Baccani, C., Capobianco, G., Fineschi, S., Focardi, M., Landini, F., Loreggia, D., Massone, G., Nicolini, G., Noce, V., Pancrazzi, M., Romoli, M., Buckley, S., O’Neill, K., Renotte, E., Servaye, J. S., and Thizy, C., "The Shadow Positioning Sensors (SPS) for formation flying metrology on-board the ESAPROBA3 mission," in [Solar Physics and Space Weather Instrumentation VI], Proc. SPIE 9604, 96040C (2015). https://doi.org/10.1117/12.2191829.

[8] Amadori, F., Capobianco, G., Loreggia, D., Casti, M., Bemporad, A., Fineschi, S., Noce, V., Zangrilli, L., Belluso, M., and Pancrazzi, M., [End-to-end numerical simulator of the Shadow Position Sensor (SPS) metrology subsystem of the PROBA-3 ESA mission], vol. 65 of INAF Technical Reports (2020). http://dx.doi.org/10.20371/INAF/TechRep/65.

[9] Noce, V., Capobianco, G., Bemporad, A., Buckley, S., Romoli, M., Casti, M., Belluso, M., Fineschi, S., Billotta, S., Loreggia, D., Thizy, C., and Napiello, L., "The Shadow Position Sensors (SPS) metrology subsystem on-board PROBA3 mission : Design and performance," in [2019 IEEE 5th International Workshop on Metrology for AeroSpace (MetroAeroSpace)], 111-115 (2019). 